\title{
The SpineBox: A Freely Available, Open-access, 3D-printed Simulator Design for Lumbar Pedicle Screw Placement
}

\author{
William Clifton $^{1}$, Aaron Damon ${ }^{1}$, Fidel Valero-Moreno ${ }^{1}$, Eric Nottmeier ${ }^{1}$, Mark Pichelmann ${ }^{1}$ \\ 1. Neurological Surgery, Mayo Clinic, Jacksonville, USA
}

Corresponding author: William Clifton, clifton.william@mayo.edu

\section{Abstract \\ Background}

The recent COVID-19 pandemic has demonstrated the need for innovation in cost-effective and easily produced surgical simulations for trainee education that are not limited by physical confines of location. This can be accomplished with the use of desktop three-dimensional (3D) printing technology. This study describes the creation of a low-cost and open-access simulation for anatomical learning and pedicle screw placement in the lumbar spine, which is termed the SpineBox.

\section{Materials and methods}

An anonymized CT scan of the lumbar spine was obtained and converted into 3D software files of the L1-L5 vertebral bodies. A computer-assisted design (CAD) software was used to assemble the vertebral models into a simulator unit in anatomical order to produce an easily prototyped simulator. The printed simulator was layered with foam in order to replicate soft tissue structures. The models were instrumented with pedicle screws using standard operative technique and examined under fluoroscopy.

\section{Results}

Ten SpineBoxes were created using a single desktop 3D printer, with accurate replication of the corticocancellous interface using previously validated techniques. The models were able to be instrumented with pedicle screws successfully and demonstrated quality representation of bony structures under fluoroscopy. The total cost of model production was under $\$ 10$.

\section{Conclusion}

The SpineBox represents the first open-access simulator for the instruction of spinal anatomy and pedicle screw placement. This study aims to provide institutions across the world with an economical and feasible means of spine surgical simulation for neurosurgical trainees and to encourage other rapid prototyping

Received 04/01/2020

Review began 04/10/2020 Review ended 04/11/2020 Published 04/20/2020

\section{() Copyright 2020}

Clifton et al. This is an open access article distributed under the terms of the Creative Commons Attribution License CC-BY 4.0., which permits unrestricted use, distribution, and reproduction in any medium, provided the original author and source are credited.

Categories: Medical Simulation, Neurosurgery, Orthopedics

Keywords: spine models, covid-19, simulation, medical education, spine surgery, lumbar spine, $3 \mathrm{~d}$ printing, threedimensional (3d) printing, neurosurgery, vertebrae

\section{Introduction}

Simulation in neurosurgical training is becoming a prominent adjunct to classical intraoperative teaching of surgical skills and classroom didactics for anatomical education [1,2]. The implementation of duty hour requirements and other tools to decrease fatigue and resident burn-out have presented a challenge to institutions in providing both adequate quantity of and quality surgical procedural experience in order to produce well-trained surgical physicians [3]. In the field of neurosurgery specifically, the rarity of cases and relatively low general incidence of neurosurgical diseases compared to other surgical subspecialties presents additional challenges to ensure adequate trainee exposure to surgical pathology [4]. Three-dimensional printing (3DP) has provided a means for recreating key points of anatomy for anatomical and procedural learning, especially in the realm of spinal surgery [5]. The feasibility of creating low-cost but high-quality 3DP simulation has been investigated, with good results [6-8]. As the number of desktop 3DP continues to move into a standard workflow for many institutions, there remains a large shortage of simulator designs that do not require purchase or extensive assembly [9].

In the current wake of the COVID-19 outbreak, the fragility of the worldwide educational platform in surgical training has been made apparent, and many trainees are unable to continue to practice surgical skills due to the massive decrease in elective case volumes and currently unspecified quarantine time [10]. Currently, the mainstay of surgical education is live operative experience, which has continued to remain 
unchanged for over a century. The recent world events have demonstrated the immediate need for research and innovation in producing broadly accessible simulators in order to maintain educational activities for surgical residents. Simulator designs for neurosurgical resident education and skills training can be developed and implemented using desktop 3D printing methods that are inexpensive and widely available [11-13]. This report describes a novel simulator design for spine anatomy and instruction of pedicle screw placement in the lumbar spine and provides a link to the Standard Tessellation Language (STL) file for immediate printing and use. This simulator can be used as an in-house or "take-home" method of instructing residents in lumbar pedicle screw placement.

\section{Materials And Methods}

Before the simulator was constructed, the desired primary learning goals of the simulator design were clearly established. This methodology has been shown to improve the teaching elements of simulators by directing learning objects to specific task-training $[14,15]$. The primary learning goal of the simulator was to instruct junior neurosurgical trainees in the anatomy and technique for pedicle screw placement in the lumbar spine. In order for this to be accomplished, the anatomical and physiologic components of the lumbar vertebrae needed to be replicated, apart from the creation of a simulated operative environment. The key components of the simulation that were included in the initial design planning were as follows: 1. representative surface anatomy of the L1-L5 vertebrae; 2. replication of the cortico-cancellous interface; 3. preservation of the natural anatomical location and direction of the lumbar pedicles at each level (e.g., lordosis); and 4. replication of an intraoperative environment with the operative field deep to a set surface level (i.e., located deep to a "skin-level" surface).

In order to produce lumbar vertebral models that adequately replicated normal surface anatomy and ultrastructure, an anonymized CT scan of the lumbar spine in an adult patient was acquired through formal IRB approval. The scan was inspected for any previous surgical defects or degenerative pathology that would interfere with the learning objectives for the simulation. The CT scan was uploaded into 3D Slicer (Slicer, v.4.0), an open-access DICOM (Digital Information and Communication in Medicine) viewing platform that allows for the creation of 3D anatomical models for 3DP and viewing. The L1-L5 vertebrae were individually selected using the threshold module, and the facet and intervertebral spaces were manually separated to produce individually segmented models of the lumbar vertebrae. This was accomplished by setting the threshold limits to 193-3,000 HU. Each lumbar vertebral model was then converted into an STL file. All five lumbar STL files were then uploaded into Meshmixer 2017 (Autodesk, San Rafael, CA), an openaccess computer-assisted design (CAD) software platform that allows for manipulation and editing of STL files in a virtual space (Video 1). The L1-L5 STL files were inspected for the preservation of the anatomical elements allowing for proper identification of the pedicle (e.g., transverse process, mammillary process, pars interarticularis). Additionally, each model was edited in order to remove unnecessary infill elements that would consume unnecessary material and printing time, and also to prevent the creation of a hollow model for programming infill for replication of the cortico-cancellous interface. The lumbar vertebrae were then arranged in anatomical order, with preservation of the lumbar lordosis [16].

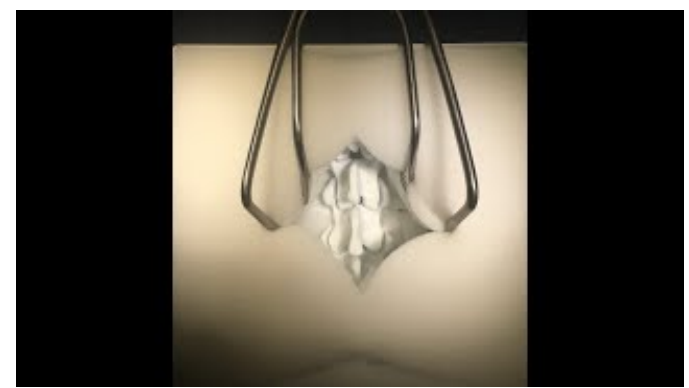

\section{VIDEO 1: Design and use of the SpineBox simulator}

View video here: https://www.youtube.com/watch?v=mhnXI2YVOEo\&t=4s

Using the Meshmixer CAD software, a virtual housing box was constructed in order to provide an operative field for the vertebral models. The X-Y dimensions of the box were adjusted to provide adequate space superiorly to the L1 vertebra and inferiorly to the L5 vertebra, and medial/lateral to the transverse processes at each level. The $\mathrm{Z}$ dimension was adjusted in order to provide coverage of the superior level over the spinous processes, thus creating a "work-in-a-hole" design for the finished simulation. The individual STL files were combined and saved as one object. The finished SpineBox STL file was then uploaded into ideaMaker (Raise3D Technologies, Irvine, CA), our laboratory's chosen software for slicing and G-code preparation. At this point in the design, the L1-L5 vertebral models were "floating" within the simulator box and thus would not be able to be printed. Using the placement of support material within the slicing software, the L1-L5 vertebral models were connected to the floor of the simulator box, thus ensuring the ability to print the entire object in one session and minimizing assembly post-production. The sliced SpineBox STL file was converted into a G-code and printed using acrylonitrile butadiene styrene (ABS) on a 


\section{Cureus}

Raise3D Pro Plus fused deposition modeling (FDM) 3D printer (Raise3D Technologies, Irvine, CA).

\section{Results}

The SpineBox was successfully printed using $1.75 \pm 0.05$-mm ABS filament through a 0.4 -mm nozzle, bed temperature of $110^{\circ} \mathrm{C}$, nozzle temperature of $265^{\circ} \mathrm{C}$, and a print speed of $55 \mathrm{~mm} / \mathrm{second}$. The total print time was 30 hours and 17 minutes. The total material expenditure was 322 grams, and the total cost of each SpineBox was $\$ 9.68$ ( $\$ 0.03$ per gram of ABS). Ten SpineBoxes were printed successfully in the initial pilot study. The dimensions of the finished print were 17.6 x 18.6 x $11.3 \mathrm{~cm}$ (Figure 1).

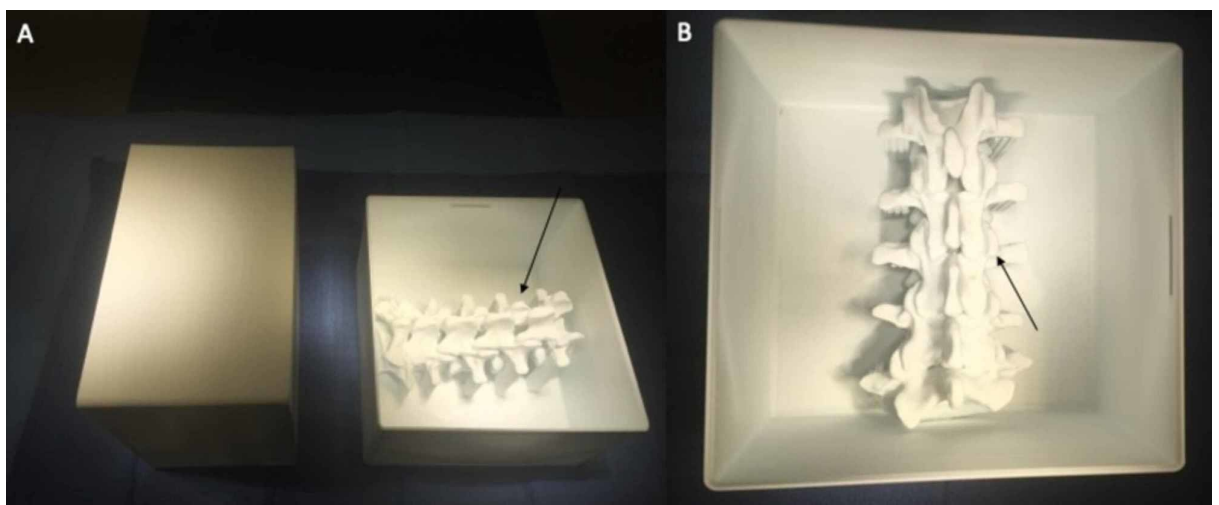

\section{FIGURE 1: SpineBox finished print}

A: the lumbar vertebral models are located beneath the surface of the top of the housing unit (arrow); B: the posterior element landmarks for pedicle screw placement can be visualized from a posterior approach in a simulated operative field (arrow)

In order to replicate soft tissue structures and create a hindrance of visualization during screw placement for increased educational value, flexible upholstery polyurethane foam sheets were acquired and cut to fit the $\mathrm{X}$ and $\mathrm{Y}$ dimensions of the simulator housing box $(17.6 \times 18.6 \mathrm{~cm})$ and layered until the foam was flush with the top limit of the box. Smaller pieces were also cut and placed within the depths of the box underneath the transverse processes of the lumbar vertebrae. After complete assembly, the foam was able to be cut with a scalpel, and retractors could be placed in order to simulate the displacement of soft tissue for operative cavity visualization (Figure 2). The total cost of upholstery foam was $\$ 0.001$ per $\mathrm{cm}^{3}$.

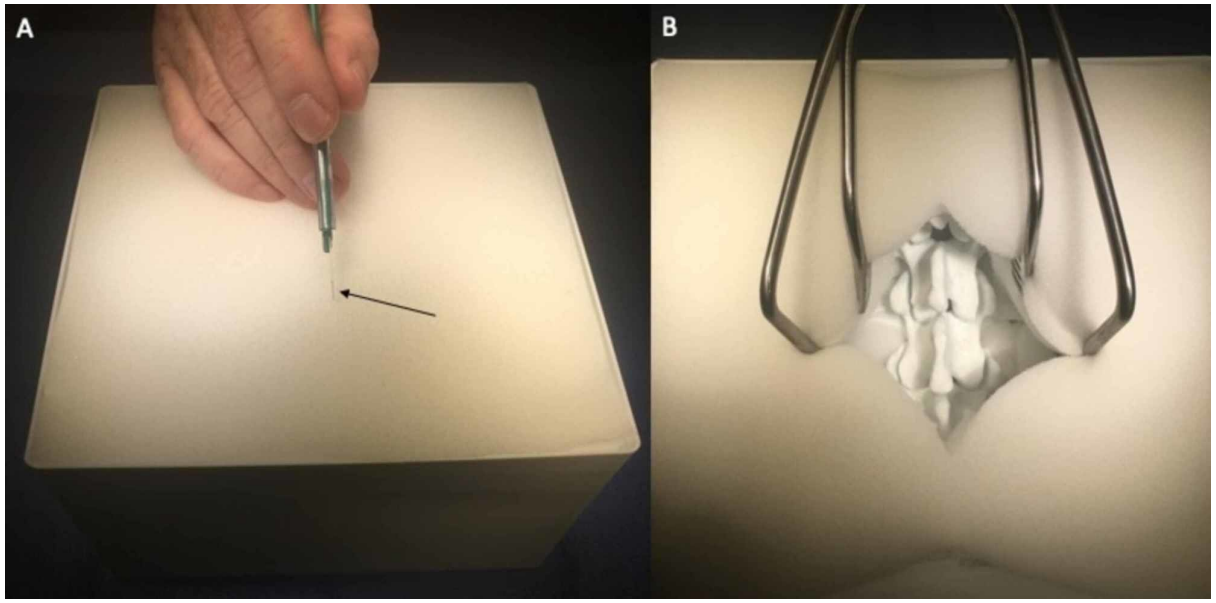

\section{FIGURE 2: The use of polyurethane foam for soft tissue simulation}

A: soft polyurethane foam is used in layers to replicate visualization hindrance of the bony posterior elements of the lumbar spine, and can be cut with a scalpel (arrow); B: retractors can be used for exposure of the lumbar spine with similar instrument-working depth, as in a live operative scenario

A total of 100 pedicle screws in 50 lumbar levels were placed within the 10 models. Five models were filled with polyisocyanate foam in order to replicate a previously validated method of simulating cancellous bone [17]. The other five models were sliced with a $25 \%$ infill in a honeycombed pattern within the vertebrae, which is another validated method of simulating vertebral cancellous bone [18]. Fluoroscopy was used to 
determine the ability of the model to demonstrate pedicle screw placement analogous to an intraoperative scenario. All pedicle screws within the models were able to be visualized with fluoroscopic localization (Figure 3). After the pedicle screw placement, the lumbar vertebral models were able to be removed from the housing unit and directly examined for breaches, enhancing the learning capabilities of the simulation.
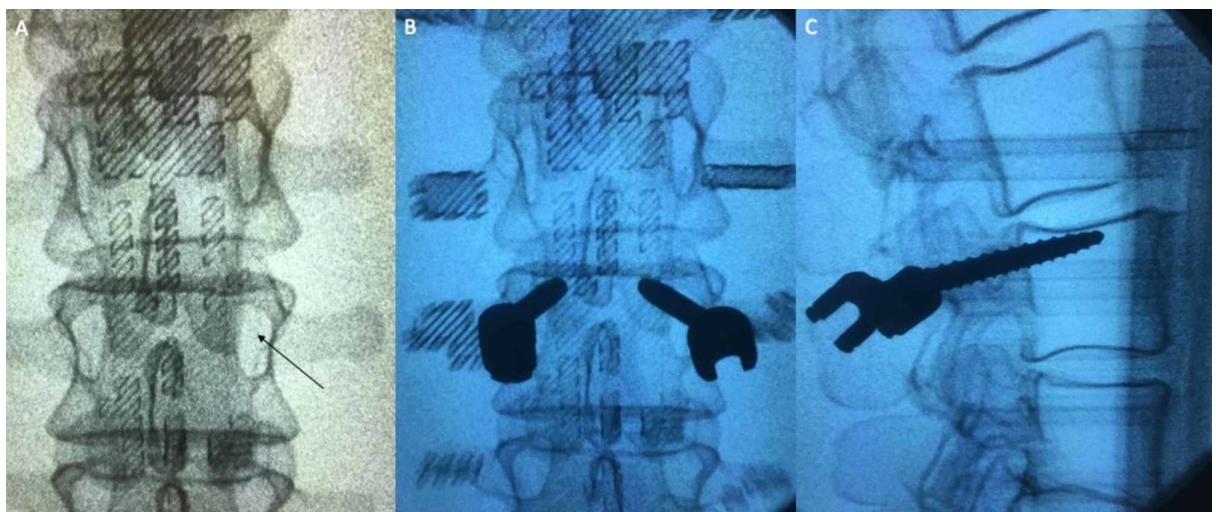

\section{FIGURE 3: Fluoroscopic investigation of the SpineBox}

A: the pedicles (arrow) of the 3D printed lumbar vertebrae can be located on fluoroscopic imaging; B and C: pedicle screws (black objects) can be seen within the model after being placed, and graded based on location for both teaching and objective skill assessment

\section{Discussion}

Spine surgical simulation has made tremendous growth and improvements in quality over the last decade and the presence of elaborate simulators in the current field has shown the ability of 3DP to create new ways of simulating spine surgery for trainees [19,20]. Still, the ability of training institutions to acquire such simulation without purchase or considerable assembly requirements remains limited [21,22]. In the current global scenario, it has become increasingly important to have access to learning tools that are mobile and independent of hospital-related conditions. The recent outbreak of COVID-19 has diminished many neurosurgical learning opportunities throughout the world due to conference cancellations, decreased operating room utility, and quarantine policies [23]. In the present landscape of spine surgical simulation, a simulator design that is easily created and portable for dissemination to neurosurgical trainees does not exist. At our institution, we have implemented the use of CAD design and 3DP to construct the SpineBox simulator, which is cost-effective, disposable, and able to replicate the cortico-cancellous interface for pedicle screw placement while providing a surgical environment that enables trainees to operate in realistic conditions with respect to instrument depth.

3D printing has been made widely available with desktop FDM 3DP models that are inexpensive and have a relatively small learning curve [24]. The common thermoplastics used for FDM printing materials have similar properties to cortical and cancellous bone and have been investigated to replicate human vertebrae for pedicle screw placement with excellent results [14,19,25]. Combining 3DP thermoplastics with dualmaterial printing or injection techniques are also distinct advantages of FDM printing for vertebral simulation compared with other 3DP methods [26]. Many teaching institutions have implemented the use of 3DP into their curriculum for anatomy and surgical education due to the relatively low cost and convenience of creating "in-house" models [20,25,27-29]. Despite these advantages, there are currently a limited number of open-access and freely available STL files for the surgical simulation that have been validated. This is partly due to industrial motivation and the potential for commercialization of simulator designs, as well as limitations in the reproducibility of construction methods for the simulators themselves. Investigators have examined the use of 3DP vertebral models placed within a foam operative cavity to simulate pedicle screw placement for the thoracolumbar spine, with excellent results in face, content, and validation [22]. The constructed simulators have shown promise for providing a quality surgical experience for neurosurgical trainees learning to place pedicle screws. Other investigators have explored fiberglass texture cervical vertebral models created through a reverse molding technique with 3DP molds placed within polyvinyl alcohol derivatives to create a cervical laminectomy simulation, with excellent results for task training and objective skill assessment [21]. Now, more than ever, innovation in 3DP simulators such as these models must continue to be investigated and produced in order to assist with the ever-growing needs of the surgical community in training the next generation of surgeons.

The SpineBox is able to replicate several validated methods of creating the cortico-cancellous interface for pedicle screw placement and to teach haptic feedback of both proper placement and breaches during pedicle access. The individual vertebral models in the simulator are designed hollow in order to allow the user to create infill gradients with their chosen slicing software before printing, or to fill with porous foams that accurately replicate the material properties of cancellous bone. Both of these methods have been previously 
validated as accurate methods of simulating cortical and cancellous bone $[12,13,17]$. The space around the vertebral models within the housing box can also be easily filled with soft foam, cotton, silicone, or other materials in order to simulate soft tissue structures that can impede visualization during screw placement, and allows the use of retractors during the simulation. The spinal canal of the vertebral models is also patent and can be filled with a Penrose drain, latex tubing, or other methods to reproduce dura and/or cerebrospinal fluid if a laminectomy simulation is desired as well.

Although this particular investigation was limited to the lumbar spine for demonstration of feasibility, our lab has created SpineBox units for cervical and thoracic levels as well, both for the practice of lateral mass and pedicle screw placement. Both of these CAD files are freely available for download and can be combined with foam in the same manner as the lumbar SpineBox model to create an in-house spine surgical simulator for teaching institutions worldwide (The SpineBox STL file can be downloaded for free at the Autodesk Online Gallery using the following link: https://bit.ly/39r2BLg).

\section{Conclusions}

Neurosurgical education and simulation in the modern era must be able to reach beyond the bounds of physical confines. The recent world crisis caused by the COVID-19 pandemic has demonstrated the uncertainty surrounding the educational programs for neurosurgical trainees and exposed a widespread need for mobile and reproducible task-trainers that are low-cost and can still deliver a quality educational experience. The SpineBox is a freely available and easily 3D-printed simulator that allows for versatility in creating a quality educational tool for lumbar spine anatomy and pedicle screw placement using previously validated techniques for replicating the cortico-cancellous interface. By the open-access dissemination of this design, our team hopes to provide institutions across the world with a cost-effective means of spine surgical simulation for neurosurgical trainees and to encourage other rapid prototyping laboratories to investigate innovative means of creating educational surgical platforms in the modern era.

\section{Additional Information}

\section{Disclosures}

Human subjects: All authors have confirmed that this study did not involve human participants or tissue. Animal subjects: All authors have confirmed that this study did not involve animal subjects or tissue. Conflicts of interest: In compliance with the ICMJE uniform disclosure form, all authors declare the following: Payment/services info: All authors have declared that no financial support was received from any organization for the submitted work. Financial relationships: All authors have declared that they have no financial relationships at present or within the previous three years with any organizations that might have an interest in the submitted work. Intellectual property info: Provisional patent based on the use of polymeric foam and 3D-printed ABS vertebral models for spine surgical simulation. Other relationships: All authors have declared that there are no other relationships or activities that could appear to have influenced the submitted work.

\section{Acknowledgements}

The authors would like to thank the B.R.A.I.N. (Biotechnology Research and Innovation Neuroscience) Laboratory for their team effort in making this work possible.

\section{References}

1. Badash I, Burtt K, Solorzano CA, Carey JN: Innovations in surgery simulation: a review of past, current and future techniques. Ann Transl Med. 2016, 4:453. 10.21037/atm.2016.12.24

2. Bullock A, Webb K: Technology in postgraduate medical education: a dynamic influence on learning? Postgrad Med J. 2015, 91:646-650. 10.1136/postgradmedj-2014-132809

3. Dimitris KD, Taylor BC, Fankhauser RA: Resident work-week regulations: historical review and modern perspectives. J Surg Educ. 2008, 65:290-296. 10.1016/j.jsurg.2008.05.011

4. Stienen MN, Netuka D, Demetriades AK, et al.: Neurosurgical resident education in Europe--results of a multinational survey. Acta Neurochir (Wien). 2016, 158:3-15. 10.1007/s00701-015-2632-0

5. Bohm PE, Arnold PM: Simulation and resident education in spinal neurosurgery . Surg Neurol Int. 2015, 6:33. 10.4103/2152-7806.152146

6. Clifton W, Nottmeier E, Damon A, Dove C, Chen SG, Pichelmann M: A feasibility study for the production of three-dimensional-printed spine models using simultaneously extruded thermoplastic polymers. Cureus. 2019, 11:e4440. Accessed: April 19, 2020: https://www.cureus.com/articles/19222-a-feasibility-study-forthe-production-of-three-dimensional-printed-spine-model.... 10.7759/cureus. 4440

7. Clifton W, Damon A, Nottmeier E, Pichelmann M: The importance of teaching clinical anatomy in surgical skills education: spare the patient, use a sim!. Clin Anat. 2020, 33:124-127. 10.1002/ca.23485

8. Clifton W, Damon A, Nottmeier E, Pichelmann M: 3-dimensionally printed biomimetic surgical simulationoperative technique of a transforaminal lumbar interbody fusion: 2-dimensional operative video (Epub ahead of print). Oper Neurosurg (Hagerstown). 2019, 10.1093/ons/opz398

9. Ratinam R, Quayle M, Crock J, Lazarus M, Fogg Q, McMenamin P: Challenges in creating dissectible anatomical 3D prints for surgical teaching. J Anat. 2019, 234:419-437. 10.1111/joa.12934

10. Adalja AA, Toner E, Inglesby TV: Priorities for the US health community responding to covid-19 (Epub ahead of print). JAMA. 2020, 10.1001/jama.2020.3413 
11. Clifton WE, Damon AC, Freeman WD: Development of a lumbar drain simulator for instructional technique and skill assessment (Epub ahead of print). Neurocrit Care. 2019, 10.1007/s12028-019-00790-9

12. Clifton W, Nottmeier E, Edwards S, Damon A, Dove C, Refaey K, Pichelmann M: Development of a novel 3D printed phantom for teaching neurosurgical trainees the freehand technique of C2 laminar screw placement. World Neurosurg. 2019, 129:e812-e820. 10.1016/i.wneu.2019.06.038

13. Clifton W, Nottmeier E, Damon A, Dove C, Pichelmann M: The future of biomechanical spine research: conception and design of a dynamic 3D printed cervical myelography phantom. Cureus. 2019, 11:e4591. Accessed: April 19, 2020: https://www.cureus.com/articles/19622-the-future-of-biomechanical-spineresearch-conception-and-design-of-a-dynamic-3.... 10.7759/cureus.4591

14. Schoenherr JR, Hamstra SJ: Beyond fidelity: deconstructing the seductive simplicity of fidelity in simulatorbased education in the health care professions. Simul Healthc. 2017, 12:117-123. 10.1097/SIH.0000000000000226

15. Selden NR, Barbaro N, Origitano TC, Burchiel KJ: Fundamental skills for entering neurosurgery residents: report of a Pacific region "boot camp" pilot course, 2009. Neurosurgery. 2011, 68:759-764. 10.1227/NEU.0b013e3182077969

16. Sparrey CJ, Bailey JF, Safaee M, et al.: Etiology of lumbar lordosis and its pathophysiology: a review of the evolution of lumbar lordosis, and the mechanics and biology of lumbar degeneration. Neurosurg Focus. 2014, 36:E1. Accessed: April 19, 2020: https://thejns.org/focus/view/journals/neurosurg-focus/36/5/articlepE1.xml. 10.3171/2014.1.FOCUS13551

17. Clifton W, Pichelmann M, Vlasak A, Damon A, ReFaey K, Nottmeier E: Investigation and feasibility of combined 3D printed thermoplastic filament and polymeric foam to simulate the cortiocancellous interface of human vertebrae. Sci Rep. 2020, 10:2912. Accessed: April 19, 2020: https://www.nature.com/articles/s41598-020-59993-2. 10.1038/s41598-020-59993-2

18. Bohl MA, Mooney MA, Repp GJ, Nakaji P, Chang SW, Turner JD, Kakarla UK: The Barrow Biomimetic Spine: fluoroscopic analysis of a synthetic spine model made of variable 3D-printed materials and print parameters. Spine (Phila Pa 1976). 2018, 43:E1368-E1375. 10.1097/BRS.0000000000002715

19. Bohl MA, McBryan S, Spear C, et al.: Evaluation of a novel surgical skills training course: are cadavers still the gold standard for surgical skills training?. World Neurosurg. 2019, 127:63-71. 10.1016/j.wneu.2019.03.230

20. Baskaran V, Štrkalj G, Štrkalj M, Di Ieva A: Current applications and future perspectives of the use of 3D printing in anatomical training and neurosurgery. Front Neuroanat. 2016, 10:69. Accessed: April 19, 2020: https://www.ncbi.nlm.nih.gov/pmc/articles/PMC4919320/. 10.3389/fnana.2016.00069

21. Weiss MY, Melnyk R, Mix D, Ghazi A, Vates GE, Stone JJ: Design and validation of a cervical laminectomy simulator using 3D printing and hydrogel phantoms. Oper Neurosurg (Hagerstown). 2020, 18:202-208. 10.1093/ons/opz129

22. Bohl MA, McBryan S, Pais D, Chang SW, Turner JD, Nakaji P, Kakarla UK: The living spine model: a biomimetic surgical training and education tool (Epub ahead of print). Oper Neurosurg (Hagerstown). 2019, 326. 10.1093/ons/opz326

23. Del Rio C, Malani PN: Covid-19-new insights on a rapidly changing epidemic (Epub ahead of print) . JAMA. 2020, 10.1001/jama.2020.3072

24. Lichtenberger JP, Tatum PS, Gada S, Wyn M, Ho VB, Liacouras P: Using 3D printing (additive manufacturing) to produce low-cost simulation models for medical training. Mil Med. 2018, 183:73-77. 10.1093/milmed/usx142

25. AbouHashem Y, Dayal M, Savanah S, Štrkalj G: The application of 3D printing in anatomy education . Med Educ Online. 2015, 20:29847. Accessed: April 19, 2020: https://www.ncbi.nlm.nih.gov/pmc/articles/PMC4609651/. 10.3402/meo.v20.29847

26. McMenamin PG, Quayle MR, McHenry CR, Adams JW: The production of anatomical teaching resources using three-dimensional (3D) printing technology. Anat Sci Edu. 2014, 7:479-486. 10.1002/ase.1475

27. Chen S, Pan Z, Wu Y, et al.: The role of three-dimensional printed models of skull in anatomy education: a randomized controlled trial. Sci Rep. 2017, 7:575. Accessed: April 19, 2020: https://www.ncbi.nlm.nih.gov/pubmed/28373643. 10.1038/s41598-017-00647-1

28. Varghese V, Krishnan V, Saravana Kumar G: Testing pullout strength of pedicle screw using synthetic bone models: is a bilayer foam model a better representation of vertebra?. Asian Spine J. 2018, 12:398-406. 10.4184/asj.2018.12.3.398

29. Clifton W, Damon A, Stein R, Pichelmann M, Nottmeier E: Biomimetic 3-dimensional-printed posterior cervical laminectomy and fusion simulation: advancements in education tools for trainee instruction. World Neurosurg. 2020, 135:308. 10.1016/j.wneu.2019.12.134 